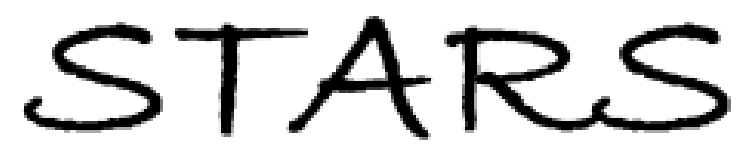

University of Central Florida

STARS

\title{
Electron irradiation-induced increase of minority carrier diffusion length, mobility, and lifetime in Mg-doped AIN/AIGaN short period superlattice
}

\author{
O. Lopatiuk-Tirpak \\ University of Central Florida \\ L. Chernyak \\ University of Central Florida \\ B. A. Borisov
}

V. V. Kuryatkov

S. A. Nikishin

Find similar works at: https://stars.library.ucf.edu/facultybib2000

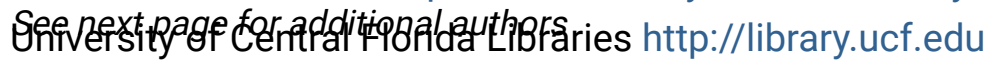

This Article is brought to you for free and open access by the Faculty Bibliography at STARS. It has been accepted for inclusion in Faculty Bibliography 2000s by an authorized administrator of STARS. For more information, please contact STARS@ucf.edu.

\section{Recommended Citation}

Lopatiuk-Tirpak, O.; Chernyak, L.; Borisov, B. A.; Kuryatkov, V. V.; Nikishin, S. A.; and Gartsman, K., "Electron irradiation-induced increase of minority carrier diffusion length, mobility, and lifetime in Mg-doped AIN/ AlGaN short period superlattice" (2007). Faculty Bibliography 2000s. 7371.

https://stars.library.ucf.edu/facultybib2000/7371

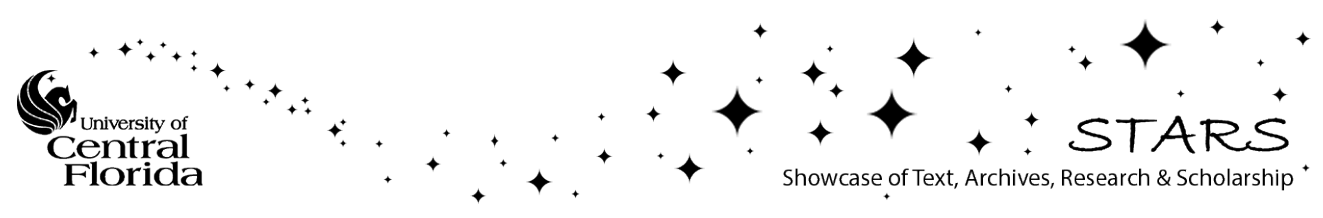


Authors

O. Lopatiuk-Tirpak, L. Chernyak, B. A. Borisov, V. V. Kuryatkov, S. A. Nikishin, and K. Gartsman 


\section{Electron irradiation-induced increase of minority carrier diffusion length, mobility, and lifetime in Mg-doped AlN / AlGaN short period superlattice}

Cite as: Appl. Phys. Lett. 91, 182103 (2007); https://doi.org/10.1063/1.2805190

Submitted: 16 August 2007. Accepted: 12 October 2007 . Published Online: 30 October 2007

O. Lopatiuk-Tirpak, L. Chernyak, B. A. Borisov, V. V. Kuryatkov, S. A. Nikishin, and K. Gartsman

\section{ARTICLES YOU MAY BE INTERESTED IN}

III-nitrides: Growth, characterization, and properties

Journal of Applied Physics 87, 965 (2000); https://doi.org/10.1063/1.371971

Refractive index of InGaN/GaN quantum well

Journal of Applied Physics 84, 6312 (1998); https://doi.org/10.1063/1.368954

GaN: Processing, defects, and devices

Journal of Applied Physics 86, 1 (1999); https://doi.org/10.1063/1.371145
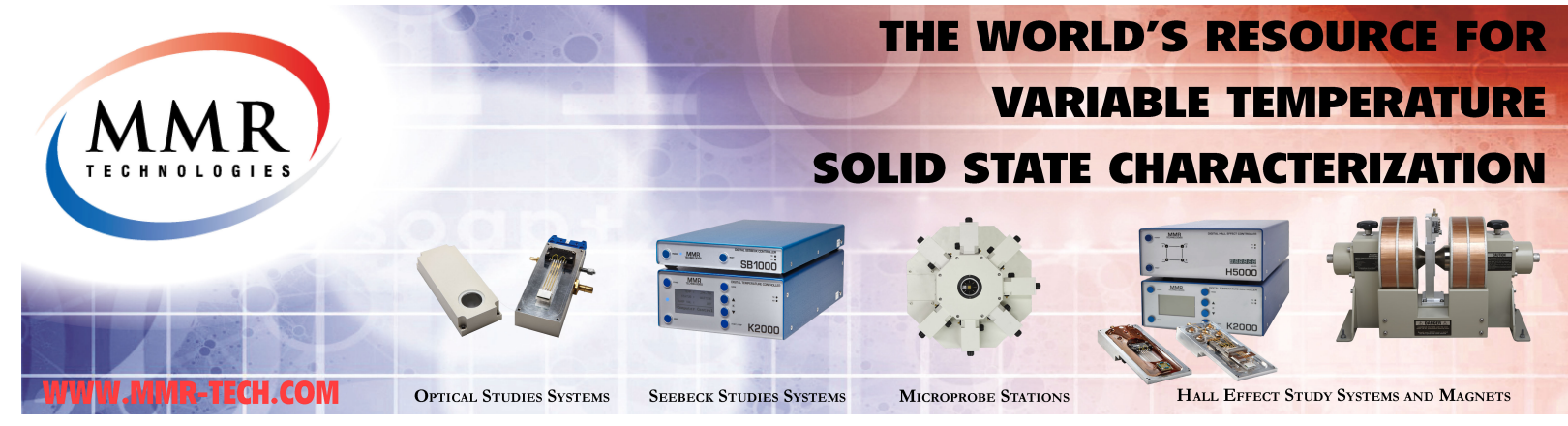


\title{
Electron irradiation-induced increase of minority carrier diffusion length, mobility, and lifetime in Mg-doped AIN/AIGaN short period superlattice
}

\author{
O. Lopatiuk-Tirpak and L. Chernyak ${ }^{\text {a) }}$ \\ University of Central Florida, Orlando, Florida 32816-2385, USA \\ B. A. Borisov, V. V. Kuryatkov, and S. A. Nikishin \\ Texas Tech University, Lubbock, Texas 79409-3102, USA \\ K. Gartsman \\ Weizmann Institute of Science, Rehovot 76100, Israel
}

(Received 16 August 2007; accepted 12 October 2007; published online 30 October 2007)

\begin{abstract}
Minority carrier diffusion length in a $p$-type $\mathrm{Mg}$-doped $\mathrm{AlN} / \mathrm{Al}_{0.08} \mathrm{Ga}_{0.92} \mathrm{~N}$ short period superlattice was shown to undergo a multifold and persistent (for at least 1 week) increase under continuous irradiation by low-energy beam of a scanning electron microscope. Since neither the diffusion length itself nor the rate of its increase exhibited any measurable temperature dependence, it is concluded that this phenomenon is attributable to the increase in mobility of minority electrons in the two-dimensional electron gas, which in turn is limited by defect scattering. Cathodoluminescence spectroscopy revealed $\sim 40 \%$ growth of carrier lifetime under irradiation with an activation energy of $240 \mathrm{meV}$. (C) 2007 American Institute of Physics. [DOI: 10.1063/1.2805190]
\end{abstract}

Over the past several years, the effects of electron irradiation on minority carrier transport have been investigated in a variety of epitaxial and bulk wide bandgap semiconductors. ${ }^{1-6}$ It was observed that, under continuous irradiation by low-energy $(10-20 \mathrm{keV})$ electron beam, the diffusion length of minority carriers $(L)$ increases severalfold in direct proportion to the duration of irradiation.

This phenomenon was correlated with the increase in lifetime of carriers in the conduction band $(\tau)$, as evidenced by the concurrent decay of luminescence intensity under irradiation. The increase in $\tau$ was attributed to the trapping of nonequilibrium electrons by the levels of deep acceptors, which are known to act as recombination centers in $\mathrm{GaN}$ and $\mathrm{ZnO}$. However, once a level captures an electron, it is effectively removed from the recombination process until the trapped electron is released. Studies of the temperature dependence of the rate of the diffusion length increase revealed that there exists considerable activation energy (several hundreds of $\mathrm{meV}$ ) for the release of the trapped electron. Therefore, as irradiation proceeds, the concentration of occupied traps goes up, leading to a decrease in the recombination rate and, consequently, to greater lifetime of nonequilibrium carriers in the conduction band. Since carrier lifetime is directly related to the diffusion length $(L=\sqrt{D \tau}$, where $D$ is the carrier diffusivity) and given the fact that the activation energy of the irradiation effects has been found to correlate to that of the dominant acceptor species, such mechanism provided a feasible explanation for the previously observed increase of the diffusion length under electron irradiation.

This explanation was also consistent with the earlier finding that carrier mobility and, therefore, the diffusivity $(D=\mu k T / q$, where $\mu$ is the mobility and $q$ is the elementary charge) remained constant under excitation by a low-energy electron beam in epitaxial $\mathrm{GaN}^{7}$ On the other hand, there exists a number of reports to suggest that the response of

\footnotetext{
${ }^{a)}$ Electronic mail: chernyak@physics.ucf.edu
}

mobility to excitation in systems with compositional inhomogeneity may be considerably different; a multifold increase in mobility with increasing concentration of nonequilibrium carriers has been demonstrated in inhomogeneous $\mathrm{Al}_{x} \mathrm{Ga}_{1-x} \mathrm{~N}$ alloys, ${ }^{8}$ AlGaN/GaN heterostructures, ${ }^{9,10}$ and GaAs/AlAs short-period superlattices (SPSLs). ${ }^{11}$

In this letter, we present the cathodoluminescence (CL) and electron beam induced current (EBIC) studies of the effects of electron irradiation on the minority carrier diffusion length in a $(\mathrm{AlN} / \mathrm{AlGaN}): \mathrm{Mg}$ SPSL. We demonstrate that while the lifetime of carriers in the conduction band does exhibit a weak increase under irradiation in agreement with earlier studies, ${ }^{1-6}$ the observed multifold growth of the diffusion length is most likely attributable to the strong dependence of mobility on carrier concentration.

The sample used in this study was grown by gas source molecular beam epitaxy with ammonia on $c$-plane sapphire using thin AlN buffer layer. The structure under investigation consisted of a $\mathrm{AlN} / \mathrm{Al}_{0.08} \mathrm{Ga}_{0.92} \mathrm{~N}$ SPSL homogeneously doped with $\mathrm{Mg}$, followed by a $20 \mathrm{~nm}$ layer of $\mathrm{GaN}: \mathrm{Mg}$. The SPSL was comprised of 400 periods, with a barrier and a well thickness of about 0.75 and $1.00 \mathrm{~nm}$, respectively, for a total of roughly $700 \mathrm{~nm}$. The average Al composition of $\sim 60 \%$ for this SPSL was determined from the zero-order $\mathrm{x}$-ray diffraction peak using Vegard's law. Hall effect measurements yielded $p$-type conductivity with hole concentration, mobility, and resistivity of $6.6 \times 10^{17} \mathrm{~cm}^{-3}$, $2.9 \mathrm{~cm}^{2} / \mathrm{V} \mathrm{s}$, and $3.3 \Omega \mathrm{cm}$, respectively. Gold Schottky contacts necessary for EBIC measurements were deposited using electron beam evaporator.

EBIC and CL measurements were conducted in situ in the Philips XL30 scanning electron microscope (SEM) under the accelerating voltage of $5 \mathrm{kV}$, corresponding to the beam penetration depth of roughly $200 \mathrm{~nm}$, assuring that the excitation is confined to the SPSL. We note that due to the low energy of the electron beam used in this study, the generation of irradiation-induced defects and structural modification of 


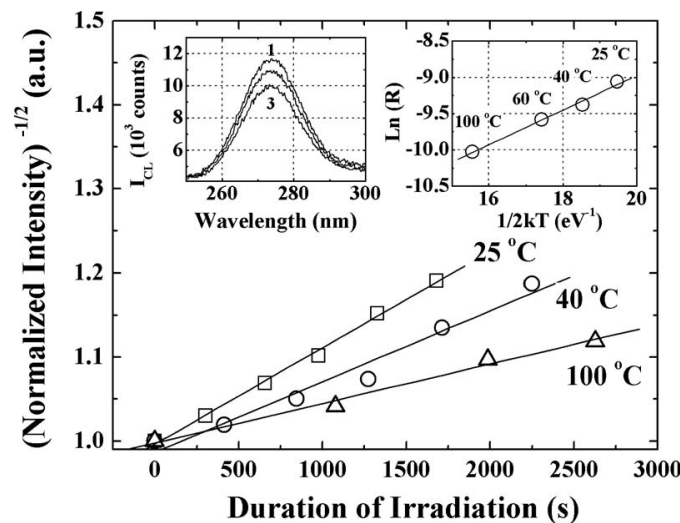

FIG. 1. The dependence of NBE CL intensity on duration of irradiation by the electron beam at several temperatures. The intensity was normalized with respect to its preirradiation value at each temperature. Left inset: Room-temperature NBE CL spectra after different intervals of continuous electron irradiation: spectrum 1 corresponds to preirradiation condition, and spectra 2 and 3 correspond to 650 and $1320 \mathrm{~s}$ of irradiation, respectively. Right inset: Temperature dependence of $R$ (see text) yielding the activation energy of $239 \pm 17 \mathrm{meV}$.

the superlattice are highly unlikely. ${ }^{5}$ EBIC measurements were performed in a linescan mode, where the induced current is measured as a function of distance of the SEM beam from the Schottky barrier (see Ref. 12 for the detailed description of EBIC method). After the initial acquisition, the beam was allowed to move repeatedly along the same line on the sample surface, while additional measurements were taken intermittently to monitor the increase of $L$. The CL measurements were conducted under the same conditions. The sample temperature was varied using a hot stage and an external temperature controller (Gatan). At each temperature, the measurements were taken from a previously unexposed location.

The experiments were started with the studies of the effect of electron irradiation on carrier lifetime using CL spectroscopy (Fig. 1). The CL spectrum shown in the left inset of Fig. 1 features a peak centered at about $270 \mathrm{~nm}(4.59 \mathrm{eV})$, which is in excellent agreement with the value expected for AlN $/ \mathrm{Al}_{0.08} \mathrm{Ga}_{0.92} \mathrm{~N}$ SPSL, with an average $\mathrm{Al}$ content of $\sim 60 \%$ and a well width of around $0.75 \mathrm{~nm} .{ }^{13,14}$ Similar to the earlier studies of other wide bandgap materials described above, the intensity of near band edge (NBE) emission (I) was shown to undergo a systematic decrease under the excitation by the electron beam. Since $I$ is inversely proportional to the nonequilibrium carrier lifetime $(\tau)$, its decay suggests that electron irradiation results in an increase of $\tau$. The latter quantity was found to increase quadratically with the duration of irradiation, as is evident from the linear increase of the inverse square root of intensity $\left(I^{-1 / 2}\right)$ in Fig. 1. It is also apparent from Fig. 1 that the rate of this increase $(R)$ diminishes with increasing temperature and is modeled with the following expression: ${ }^{15}$

$$
R=R_{0} \exp \left(\frac{\Delta E_{A}}{2 k T}\right),
$$

where $R_{0}$ is a scaling constant, $k$ is Boltzmann's constant, $T$ is the sample temperature, and $\Delta E_{A}$ is the activation energy for irradiation-induced effect. The fit of $R$ with Eq. (1), shown in the right inset of Fig. 1, yielded an activation energy of $239 \pm 17 \mathrm{meV}$ and is likely related to the activation

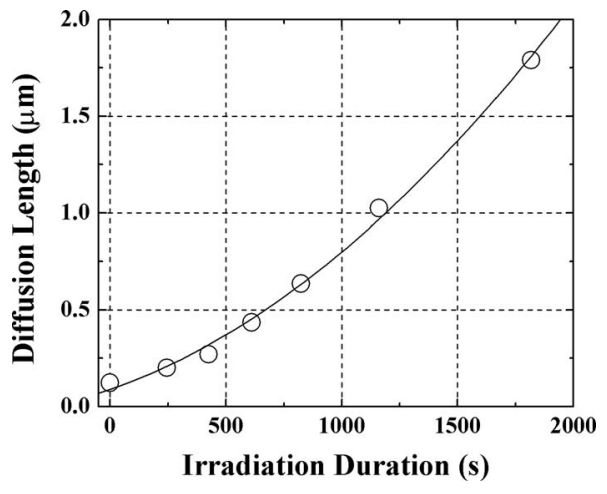

FIG. 2. Increase of minority carrier diffusion length under electron irradiation at room temperature.

energy of $\mathrm{Mg}$ acceptor. This value is in good agreement with $\mathrm{Mg}$ activation energy in AlN/ $\mathrm{Al}_{0.08} \mathrm{Ga}_{0.92} \mathrm{~N}$ SPSLs. ${ }^{16}$

The behavior of carrier lifetime summarized in Fig. 1 is consistent with the mechanism proposed earlier to explain the irradiation-induced effects observed in epitaxial GaN:Mg (see introduction above). On the other hand, a quadratic increase in carrier lifetime implies that minority carrier diffusion length should exhibit linear dependence on the duration of irradiation, since $L$ is proportional to $\tau^{1 / 2}$. However, as Fig. 2 demonstrates, the relationship between $L$ and irradiation time is clearly superlinear and can be modeled with a second-order polynomial. Given additional observations that (i) the nearly 15 -fold increase in the value of $L$ is inconsistent with a mere $40 \%$ drop in NBE CL intensity for similar irradiation times and (ii) neither $L$ nor the rate of its increase under electron irradiation exhibit any discernable temperature dependence common to bulk and epitaxial semiconductors, ${ }^{12,15}$ we conclude that in the investigated sample, this parameter is determined mainly by the mobility of minority electrons, and that the mobility, in turn, is limited by defect scattering, rather than by phonon interactions.

This finding is in contrast to our prior studies of bulk and thin-film semiconductors under electron irradiation, where the increase of $L$ was explained by the increase in carrier lifetime due to trapping of nonequilibrium electrons on deep acceptor levels. ${ }^{1-6}$ In the present case, however, it should be stressed that while the increase of $\tau$ is consistent with the trapping on nonionized $\mathrm{Mg}$ centers [as evidenced by the activation energy determined from Eq. (1)], the behavior of $L$ under irradiation is clearly dominated by a different process (see discussion below) that does not appear to be associated with the trapping on deep defect levels.

The increase of majority carrier mobility under photoexcitation has been observed earlier and was correlated with the increase in majority carrier concentration $(n)^{8,9,11,17,18}$ The mobility was shown to scale with $n^{\alpha}$, although the magnitude of $\alpha$ appears to depend strongly on materials' properties and experimental conditions such as temperature.

In order to demonstrate the dependence of $L$ on the concentration of excitation-generated carriers, EBIC measurements were performed as a function of current absorbed in the sample from the SEM beam. The absorbed current was varied by adjusting the beam current and was measured by coupling the ground of the SEM to the input of a picoammeter (Keithley).

The fact that minority electron density is linearly related to the absorbed current is confirmed by the response of $\mathrm{CL}$ 


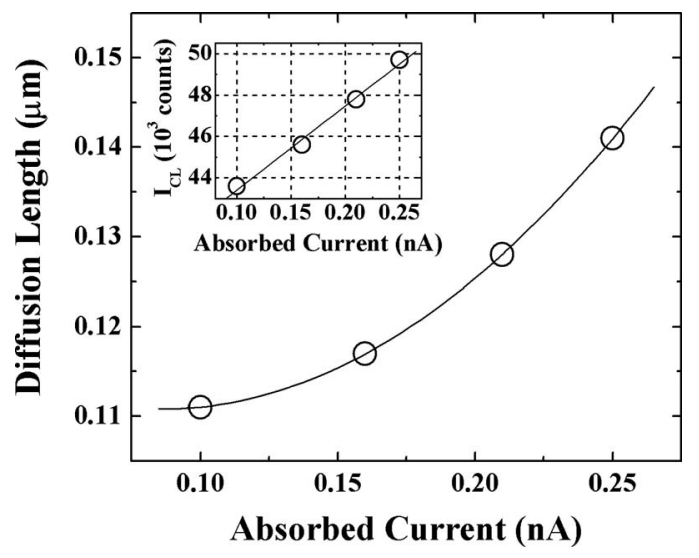

FIG. 3. Dependence of minority carrier diffusion length on the current absorbed in the sample from the SEM beam. Inset: Influence of absorbed current on the total CL intensity.

intensity shown in the inset of Fig. 3. This is because, while the recombination rate (i.e., intensity) is proportional to the product of densities of both carrier types $\left(I \propto n_{e} n_{p}\right.$, where $n_{e}$ and $n_{p}$ are electron and hole densities, respectively), the change in minority carrier concentration has a much greater impact, since for $p$-type material, $n_{p}+\Delta n_{p} \approx n_{p}$.

Our finding that during electron irradiation, $L$ varies quadratically with the absorbed current [i.e., minority electron density (see Fig. 3)] further supports the hypothesis that the irradiation-induced increase of $L$ occurs due the concentration-dependent growth of minority electron mobility. It should be noted that while the values of $\alpha$ reported in literature for majority carriers range from 0.3 to $1.6,{ }^{8,18}$ the quadratic dependence of $L$ on $n_{e}$ (see Fig. 3) implies that in the case of our experiments the mobility of minority carriers changes as $n_{e}^{4}$, since $L \propto \sqrt{\mu}$. (Although $L$ is also dependent on $\tau$, the change in the latter quantity is insignificant compared to the factor of 10 increase of the former). It has been suggested that the carrier concentration dependence of mobility can be explained by the increase in mean energy of electrons in a two-dimensional electron gas as the population of the potential well grows, resulting in a more effective screening and in a reduction in impurity and defect scattering. ${ }^{10}$

Finally, it should be noted that $L$ was found to persist at the elevated value for at least 1 week, suggesting a long-lasting nature of carrier redistribution due to electron irradiation.
In summary, it has been shown that in a Mg-doped AlN/ $\mathrm{Al}_{0.08} \mathrm{Ga}_{0.92} \mathrm{~N}$ SPSL structure, exposure to low-energy electron beam results in a considerable increase of minority carrier diffusion length. While a moderate growth of carrier lifetime has been observed, the bulk of the diffusion length response to irradiation is attributed to the growth in minority carrier mobility.

This work was supported in part by the National Science Foundation (ECS 0422604, ECS-0304224, and ECS0609416), THECB-ARP-003644-0014-2006, the American Chemical Society Petroleum Research Fund (40501-AC10), NATO Science for Peace program (SfP 981939), and the J. F. Maddox Foundation.

${ }^{1}$ W. Burdett, O. Lopatiuk, L. Chernyak, M. Hermann, M. Stutzmann, and M. Eickhoff, J. Appl. Phys. 96, 3556 (2004).

${ }^{2}$ L. Chernyak, A. Osinsky, V. Fuflyigin, and E. F. Schubert, Appl. Phys. Lett. 77, 875 (2000).

${ }^{3}$ O. Lopatiuk, W. Burdett, L. Chernyak, K. P. Ip, Y. W. Heo, D. P. Norton, S. J. Pearton, B. Hertog, P. P. Chow, and A. Osinsky, Appl. Phys. Lett. 86, 012105 (2005).

${ }^{4}$ O. Lopatiuk, L. Chernyak, A. Osinsky, and J. Q. Xie, Appl. Phys. Lett. 87, 214110 (2005).

${ }^{5}$ O. Lopatiuk, A. Osinsky, A. Dabiran, K. Gartsman, I. Feldman, and L. Chernyak, Solid-State Electron. 49, 1662 (2005).

${ }^{6}$ O. Lopatiuk-Tirpak, L. Chernyak, F. X. Xiu, J. L. Liu, S. Jang, F. Ren, S. J. Pearton, A. Osinsky, and P. Chow, J. Appl. Phys. 100, 086101 (2006).

${ }^{7}$ S. Nakamura, M. Senoh, and T. Mukai, Jpn. J. Appl. Phys., Part 2 30, L1708 (1991).

${ }^{8}$ K. C. Zeng, J. Y. Lin, and H. X. Jiang, Appl. Phys. Lett. 76, 1728 (2000).

${ }^{9}$ J. Z. Li, J. Li, J. Y. Lin, and H. X. Jiang, MRS Internet J. Nitride Semicond. Res. 5, W11.12 (2000).

${ }^{10}$ J. Z. Li, J. Y. Lin, H. X. Jiang, M. A. Khan, and Q. Chen, J. Vac. Sci. Technol. B 15, 1117 (1997).

${ }^{11}$ F. Bosc, J. Sicart, J. L. Robert, and R. Piotrzkowski, J. Appl. Phys. 88, 1515 (2000).

${ }^{12}$ L. Chernyak, A. Osinsky, H. Temkin, J. W. Yang, Q. Chen, and M. A. Khan, Appl. Phys. Lett. 69, 2531 (1996).

${ }^{13}$ M. Holtz, I. Ahmad, V. V. Kuryatkov, B. A. Borisov, G. D. Kipshidze, A. Chandolu, S. A. Nikishin, and H. Temkin, GaN and Related Alloys, MRS Symposia Proceedings No. 798 (Materials Research Society, Pittsburg, 2004), p. Y1.9.1.

${ }^{14}$ S. A. Nikishin, M. Holtz, and H. Temkin, Jpn. J. Appl. Phys., Part 1 44, 7221 (2005)

${ }^{15}$ M. Eckstein and H. U. Habermeier, J. Phys. IV 1, 23 (1991).

${ }^{16}$ V. Kuryatkov, K. Zhu, B. Borisov, A. Chandolu, I. Gheriasou, G. Kipshidze, S. N. G. Chu, M. Holtz, Yu. Kudryavtsev, R. Asomoza, S. A. Nikishin, and H. Temkin, Appl. Phys. Lett. 83, 1319 (2003).

${ }^{17}$ V. Kazukauskas, G. Kuhnel, and W. Siegel, Appl. Phys. Lett. 70, 1751 (1997).

${ }^{18}$ E. H. Hwang and S. Das Sarma, Phys. Rev. B 75, 073301 (2007). 